Unbound Natural Organic Matter Competes with Nanoparticles for Internalization Receptors during Cell Uptake

6 Ya-Tong Zhao ${ }^{\dagger}$, Shuai Yan*, Bin Huang ${ }^{\dagger}$, Liuyan Yang ${ }^{\dagger}$, Hong-ming Ding*, , Ping Wang*, Ai7 Jun Miao*,†

10 State Key Laboratory of Pollution Control and Resource Reuse, School of the Environment,

11 Nanjing University, Nanjing, Jiangsu Province, 210046, China PRC

12 Collaborative Innovation Center for Biomedical Engineering, Wuhan National Laboratory for

13 Optoelectronics, Huazhong University of Science and Technology, Wuhan, Hubei Province,

14 430074, China PRC

$15{ }^{\S}$ Center for Soft Condensed Matter Physics and Interdisciplinary Research, School of Physical

16 Science and Technology, Soochow University, Suzhou, Jiangsu Province, 215006, China PRC

17

$18 *$ Corresponding authors:

19 H.-M. Ding, dinghm@suda.edu.cn (email); A.-J. Miao, miaoaj@nju.edu.cn (email) 


\section{Coarse-Grained Modeling}

Figure S6 illustrates the coarse-grained modeling of different components (HemNPs,

22 NOM, and cell membrane) in the simulation. Each HemNP was fabricated by arranging beads on an fcc lattice (lattice constant $\alpha=0.50 \mathrm{~nm}$ ) into a sphere with a diameter of $10 \mathrm{~nm}$. All beads

24 in each nanoparticle moved as a rigid body and the beads on the nanoparticle surface served as ligands. A simplified model of the fulvic acid (FA) monomer was used as the representative

26 NOM molecule in the simulation. ${ }^{[1,2]}$ It was composed of a specific portion able to interact with the receptors and a non-specific portion that was relatively inert. The fraction of charged beads was set at 0.25 , given that carboxylic acid is a weak electrolyte. The cell membrane was represented by lipid bilayers made up of self-assembled charge-neutral lipid molecules, consisting of a head group with four connected hydrophilic beads and two tails with three hydrophobic beads each. The first head bead in the head group of the lipid molecule carried a charge of $+\mathrm{e}$, the second head bead a charge of $-\mathrm{e}$, and the remaining two beads were uncharged. ${ }^{[3]}$ To mimic the anionic molecules on the cell surface, $5 \%$ of the lipid molecules in

34 the simulation were negatively charged.$^{[4,5]}$ For this purpose, a non-charged hydrophilic bead replaced the first positively charged bead in the lipid molecules. ${ }^{[5]}$ Additionally, a simplified model (receptor-like lipid) was used to simulate membrane receptors, with the receptor-like

37 lipid having the same conformation as the aforementioned lipid molecules. ${ }^{[5,6]}$ However, as the 38 first two head beads were uncharged they were able to interact with the HemNP and the NOM via soft Lennard-Jones potentials. ${ }^{[5,7]}$

\section{REFERENCES}


41 [1] Stevenson, F. J. Humus chemistry: genesis, composition, reactions. John Wiley \& Sons: 1994.

43

44

45

46

47

48

49

50

51

52

[2] Wang, Z.; Quik, J. T. K.; Song, L.; Wouterse M.; Peijnenburg, W. J. G. M. Dissipative particle dynamic simulation and experimental assessment of the impacts of humic substances on aqueous aggregation and dispersion of engineered nanoparticles. Environ. Toxicol. Chem. 2018, 37, 1024-1031.

[3] Izvekov, S.; Voth, G. A. A multiscale coarse-graining method for biomolecular systems. J. Phys. Chem. B 2005, 109, 2469-2473.

[4] Jobin, M. L.; Alves, I. D. On the importance of electrostatic interactions between cell penetrating peptides and membranes: A pathway toward tumor cell selectivity. Biochimie 2004, 107, 154-159.

[5] Ding, H. M.; Ma, Y. Q. Design maps for cellular uptake of gene nanovectors by computer simulation. Biomaterials 2013, 34, 8401-8407.

[6] Shi, X. H.; Bussche, A. V. D.; Hurt, R. H.; Kane, A. B.; Gao, H. J. Cell entry of onedimensional nanomaterials occurs by tip recognition and rotation. Nat. Nanotechnol. 2011, 6, 714-719.

[7] Yang K.; Ma Y. Q. Computer simulation of the translocation of nanoparticles with different shapes across a lipid bilayer. Nat. Nanotechnol. 2010, 5, 579-583. 

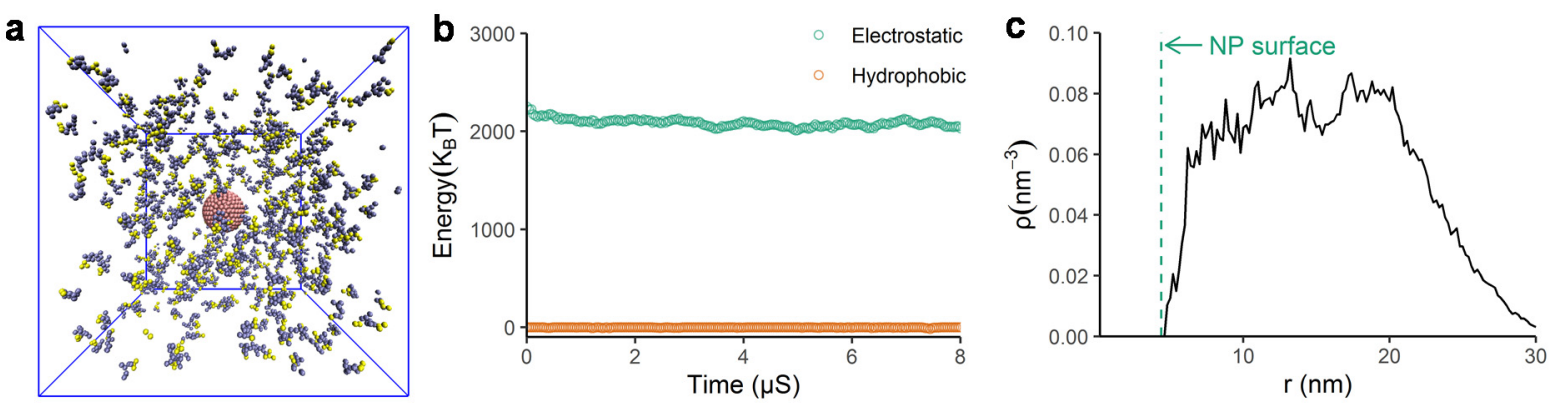

61 Figure S1. Self-assembly of HemNPs and NOM molecules in water. (a) The final snapshot

62 of the HemNPs-NOM system. Blue lines mark the borders of the simulation box. (b) A plot of

63 the evolution over time of the interaction energy (including electrostatic and hydrophobic

64 interactions between HemNPs and NOM) during the self-assembly process in the simulation

65 shows that electrostatic repulsion was the dominant force between HemNPs and NOM. Short-

66 distance forces such as hydrophobic and steric interactions were negligible. (c) An analysis of

67 the radial distribution of NOM molecules around the HemNPs (number of NOM molecules per

$68 \mathrm{~nm}^{3}$ ) shows no NOM adsorption on HemNPs. The dark green dashed line represents the

69 HemNP surface.

70 


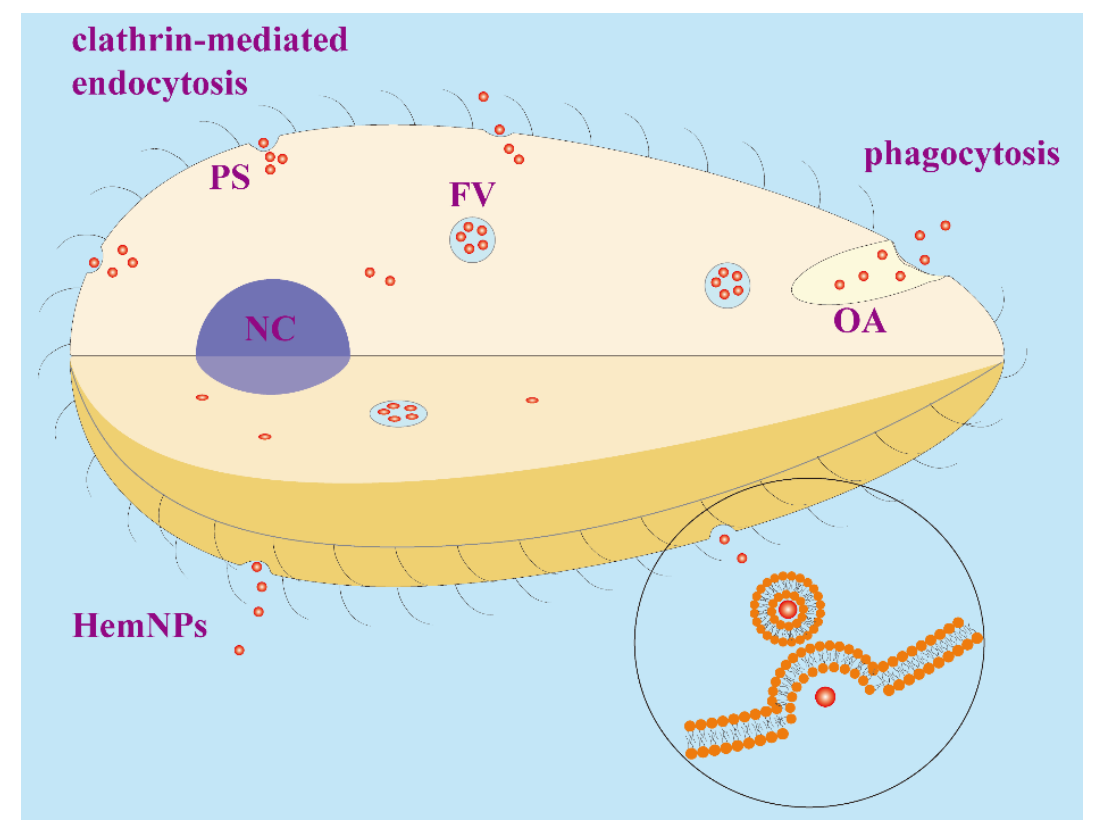

72 Figure S2. Anatomical image of Tetrahymena thermophila together with the

73 internalization mechanisms of HemNPs. In T. thermophila, internalization can occur through

74 either the oral cavity (OA) or the parasomal sacs (PS) located anterior to each of the $\sim 500-600$

75 ciliary basal bodies arranged in 17-21 longitudinal rows across the whole membrane. The OA

76 internalizes HemNPs through phagocytosis into the food vacuoles (FV). The PS takes up

77 HemNPs through clathrin-mediated endocytosis into the cytoplasm. NC means nucleus. 

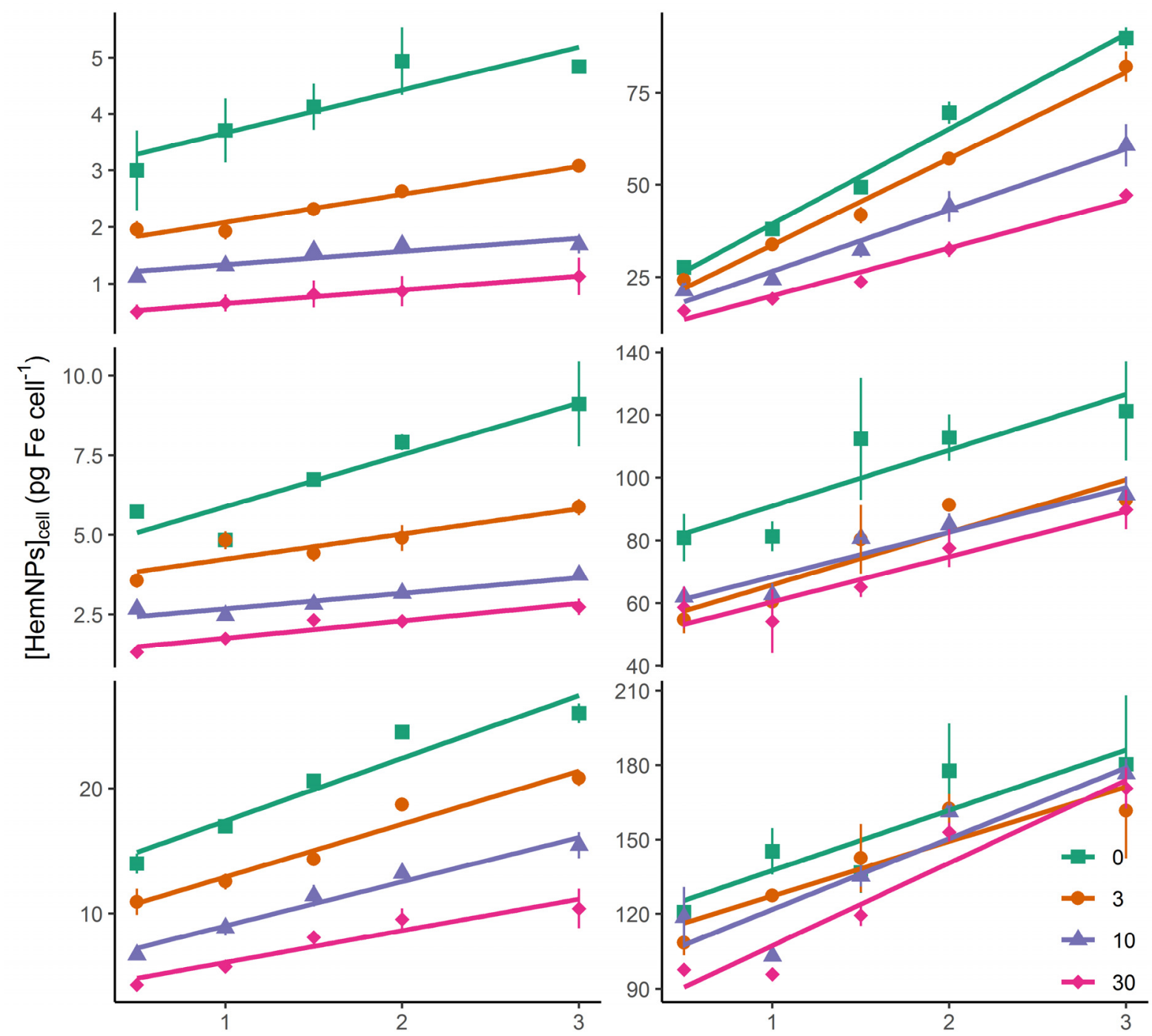

Exposure time (h)

Figure S3. Uptake kinetics of HemNPs in the presence of different concentrations of fulvic

80 acid (FA). The accumulation of HemNPs in Tetrahymena thermophila ([HemNPs]cell) at

81 different concentrations of HemNPs in the experimental medium [(a) $1 \mathrm{mg} \mathrm{Fe} \mathrm{L}^{-1}$, (b) $3 \mathrm{mg} \mathrm{Fe}$

$82 \mathrm{~L}^{-1}$, (c) $10 \mathrm{mg} \mathrm{Fe} \mathrm{L}^{-1}$, (d) $30 \mathrm{mg} \mathrm{Fe} \mathrm{L}^{-1}$, (e) $100 \mathrm{mg} \mathrm{Fe} \mathrm{L}^{-1}$, and (f) $300 \mathrm{mg} \mathrm{Fe} \mathrm{L}^{-1}$ ] increased

83 linearly with increasing exposure time. The $\mathrm{FA}\left(0,3,10\right.$, and $\left.30 \mathrm{mg} \mathrm{C} \mathrm{L}^{-1}\right)$ induced decrease in

$84[\text { HemNPs }]_{\text {cell }}$ was concentration-dependent. The data are presented as the mean \pm s.d. $(\mathrm{n}=3)$. 


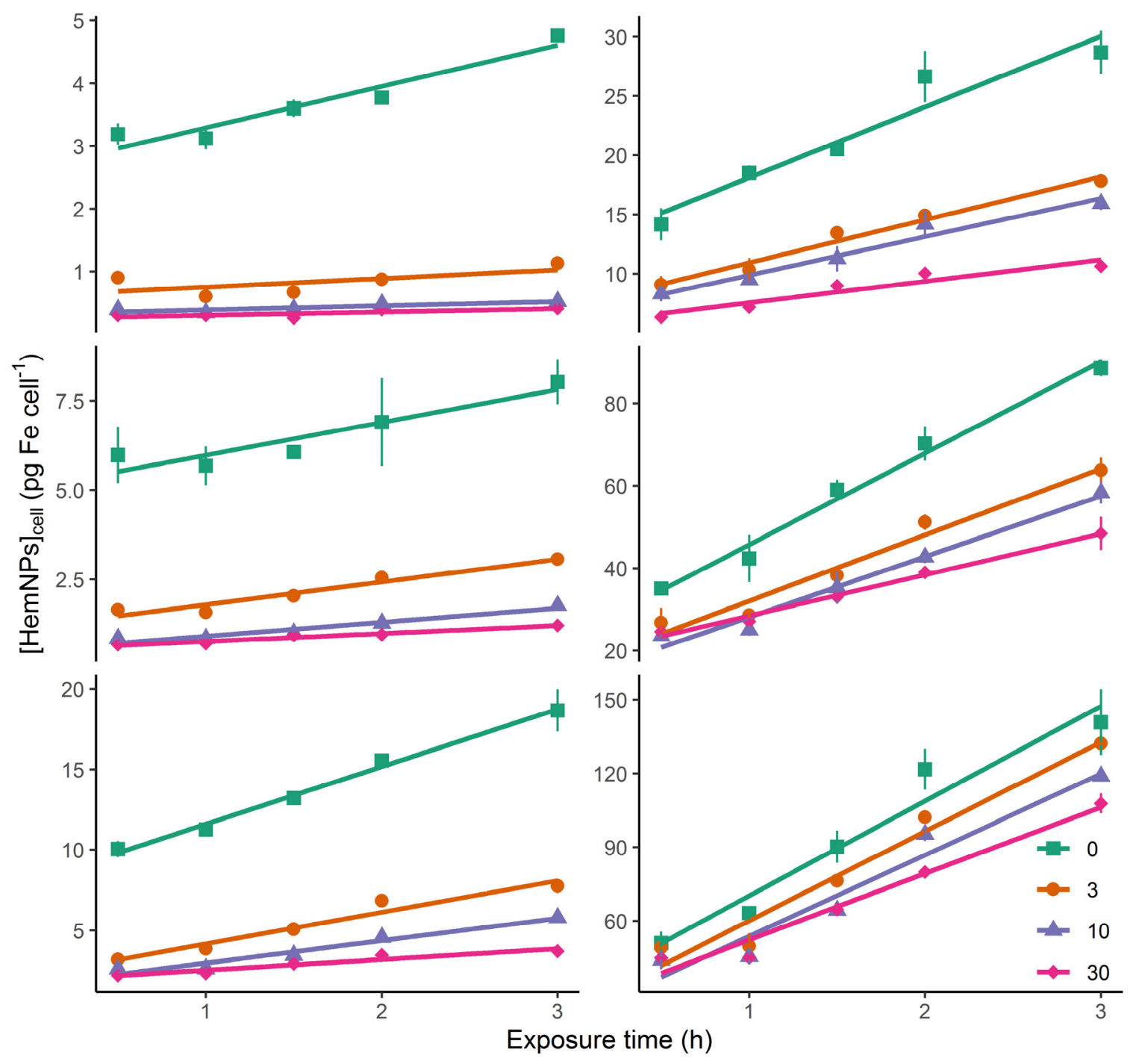

87 Figure S4. Uptake kinetics of HemNPs in the presence of different concentrations of

88 humic acid (HA). The accumulation of HemNPs in T. thermophila ([HemNPs]cell) at different

89 concentrations of HemNPs in the experimental medium [(a) $1 \mathrm{mg} \mathrm{Fe} \mathrm{L}^{-1}$, (b) $3 \mathrm{mg} \mathrm{Fe} \mathrm{L}^{-1}$, (c)

$9010 \mathrm{mg} \mathrm{Fe} \mathrm{L}^{-1}$, (d) $30 \mathrm{mg} \mathrm{Fe} \mathrm{L}{ }^{-1}$, (e) $100 \mathrm{mg} \mathrm{Fe} \mathrm{L}^{-1}$, and (f) $300 \mathrm{mg} \mathrm{Fe} \mathrm{L}^{-1}$ ] increased linearly

91 with exposure time. The HA $\left(0,3,10\right.$, and $\left.30 \mathrm{mg} \mathrm{C} \mathrm{L}^{-1}\right)$ induced decrease in [HemNPs]cell was

92 concentration-dependent. The data are presented as the mean \pm s.d. $(n=3)$. 


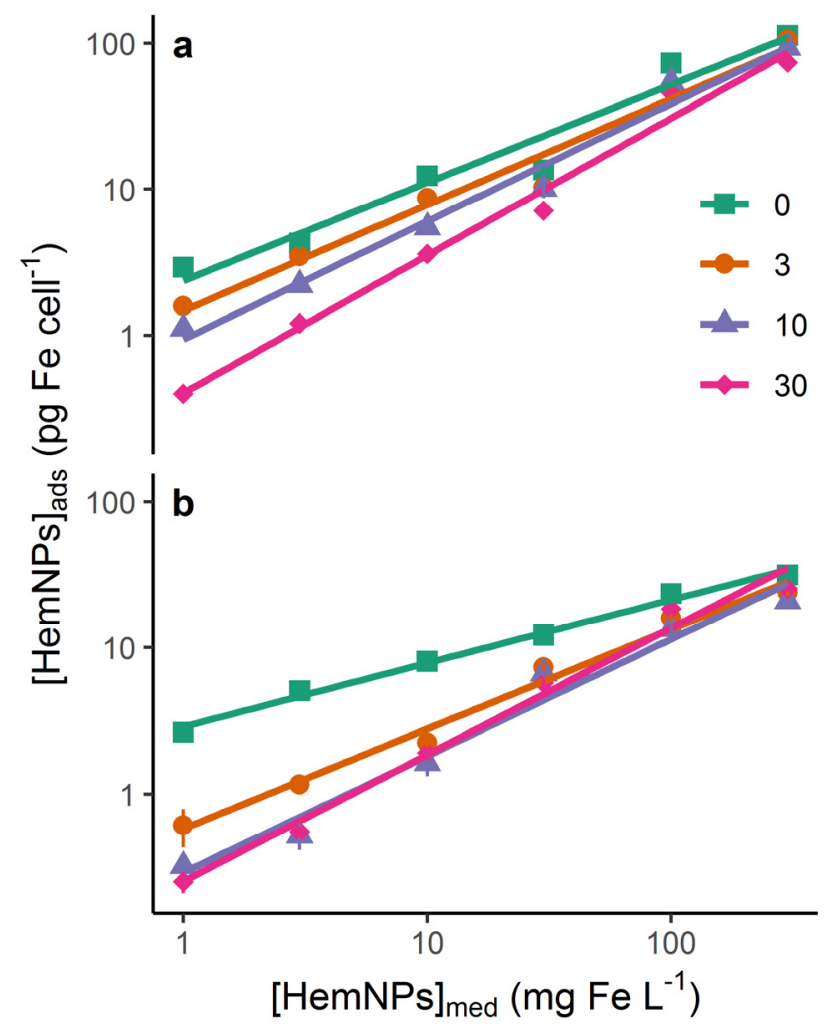

95 Figure S5. Cell surface adsorption of HemNPs. The amount of HemNPs adsorbed on the cell

96 surface of $T$. thermophila ([HemNPs $]_{\text {ads }}$ ) correlated linearly with the concentration of HemNPs

97 in the experimental medium ([HemNPs $\left.]_{\mathrm{med}}\right)$ on a log-scale. Both (a) FA and (b) HA (0, 3, 10,

98 and $30 \mathrm{mg} \mathrm{C} \mathrm{L}^{-1}$ ) induced a concentration-dependent decrease in [HemNPs]ads. Inhibition was

99 stronger at lower [HemNPs]med and in the presence of HA. The data are presented as the mean

$100 \pm$ s.d. $(\mathrm{n}=3)$.

101 


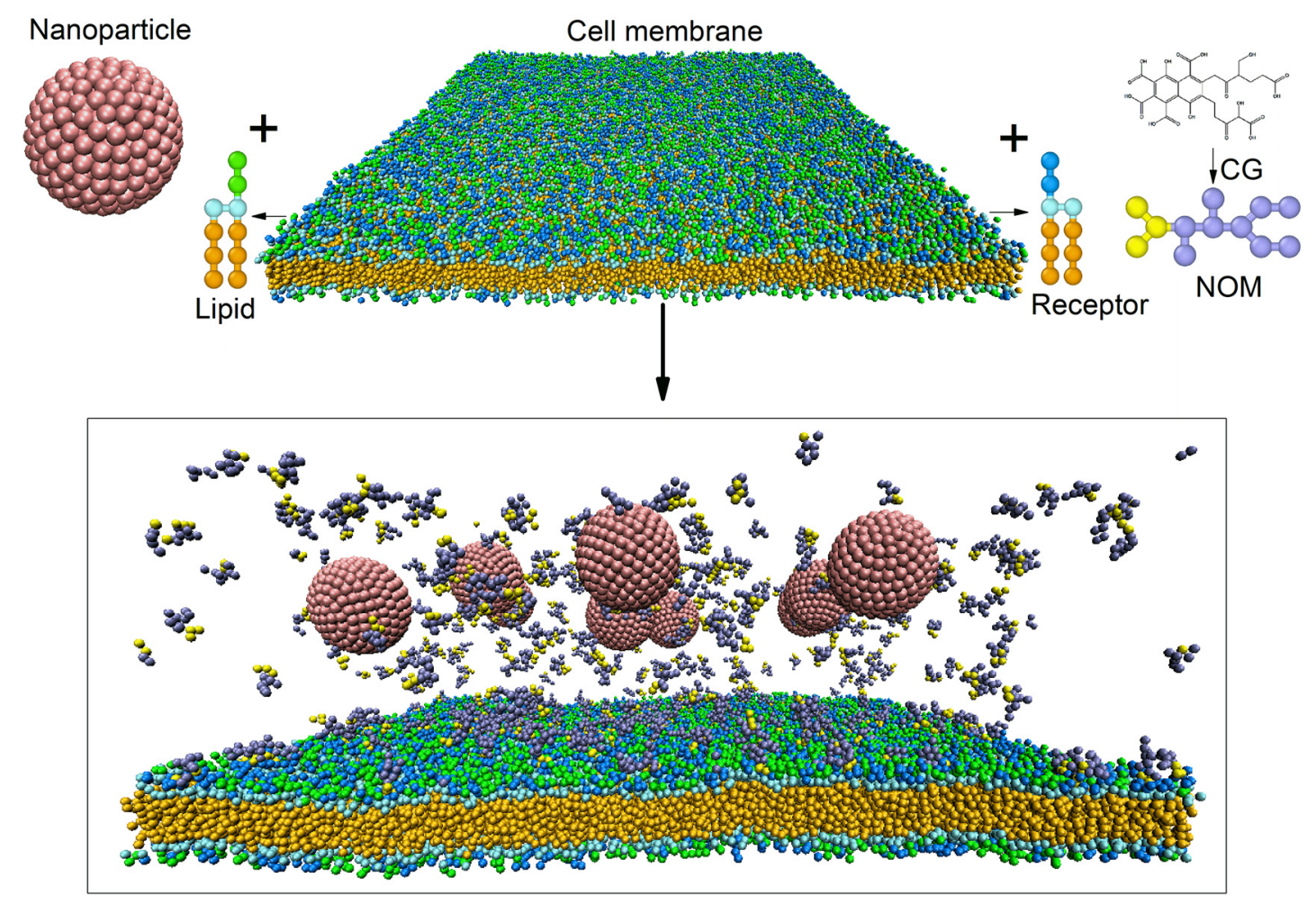

103 Figure S6. Schematic illustration of the coarse-grained (CG) models in the dissipative

104 particle dynamic simulation. HemNP beads are shown in pink. The NOM molecule is

105 composed of specific and non-specific blocks, represented by yellow and ice-blue beads,

106 respectively. The cell membrane is composed of lipids and receptors: green beads represent the

107 charged part of the lipid head, and blue beads the receptor head; lime-green beads represent the

108 charge-neutral part of the lipid and receptor head, and orange beads the tails of lipids and

109 receptors. For clarity, water molecules and ions are not shown. 


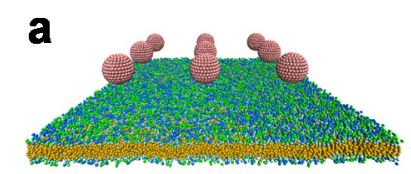

$0.4 \mu \mathrm{s}$

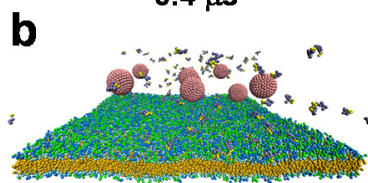

$0.8 \mu \mathrm{s}$

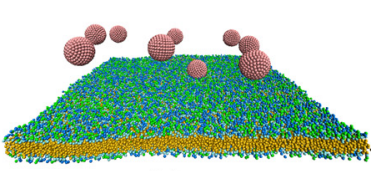

$4.8 \mu \mathrm{s}$

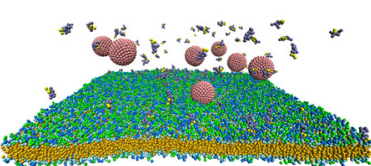

$4.4 \mu \mathrm{s}$

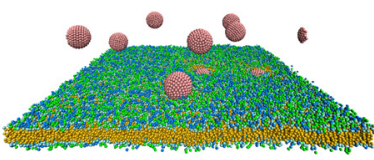

$15.0 \mu \mathrm{s}$

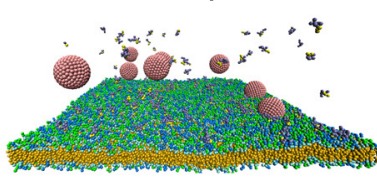

$15.0 \mu \mathrm{s}$

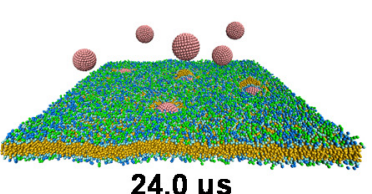

$24.0 \mu \mathrm{s}$

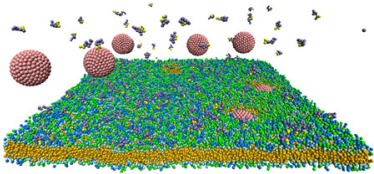

$24.0 \mu \mathrm{s}$

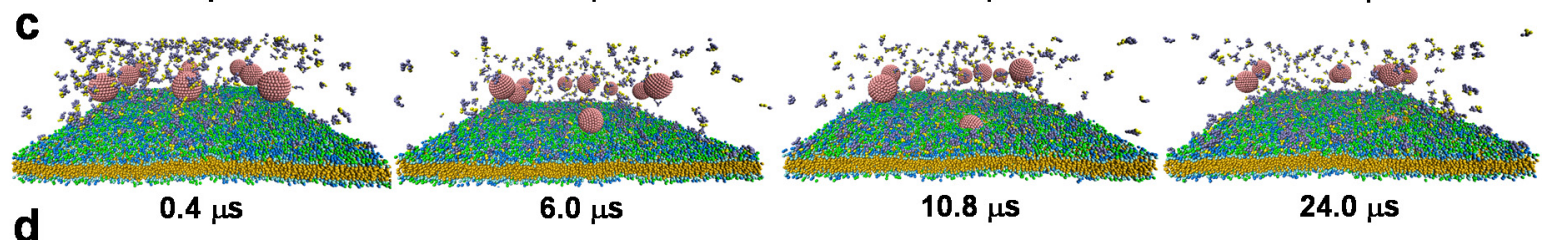

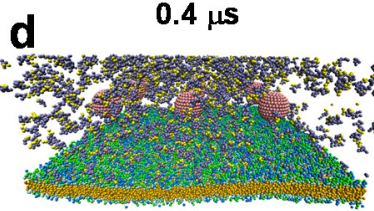

$0.6 \mu \mathrm{s}$

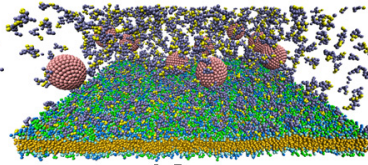

$4.8 \mu \mathrm{S}$

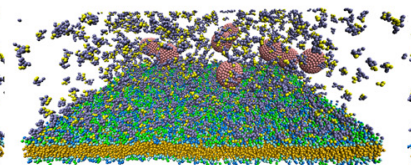

$12.0 \mu \mathrm{s}$

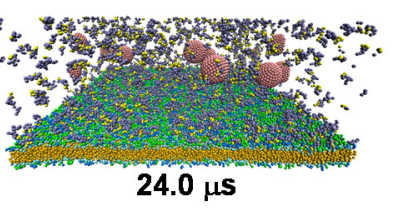

113 Figure S7. Time sequence of the dissipative particle dynamic simulation. The snapshots

114 show the typical processes in the interaction of HemNPs with the cell membrane in the absence

115 and presence of NOM: (a) No NOM, (b) NOM/NP=12, (c) $\mathrm{NOM} / \mathrm{NP}=40$, and (d)

$116 \mathrm{NOM} / \mathrm{NP}=120$. The number of HemNPs in the simulation was fixed at nine. The number of

117 HemNPs adsorbed on the cell membrane increased with time whereas adsorption decreased as

118 the NOM/NP ratio increased.

119 


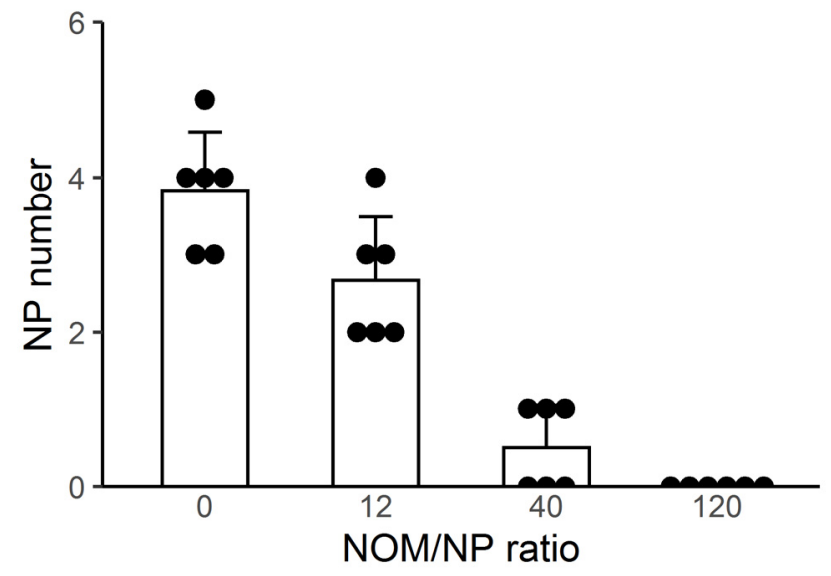

121 Figure S8. Summary of the dissipative particle dynamic simulation results for the number

122 of HemNPs adsorbed on the cell membrane. The average number of membrane-adsorbed

123 HemNPs (column) decreased as the NOM/NP ratio increased. Six simulations were performed

124 for each ratio, and the respective results were plotted as the scattered points. The data are

125 presented as the mean \pm s.d. ( $\mathrm{n}=6$ independent simulations).

126 


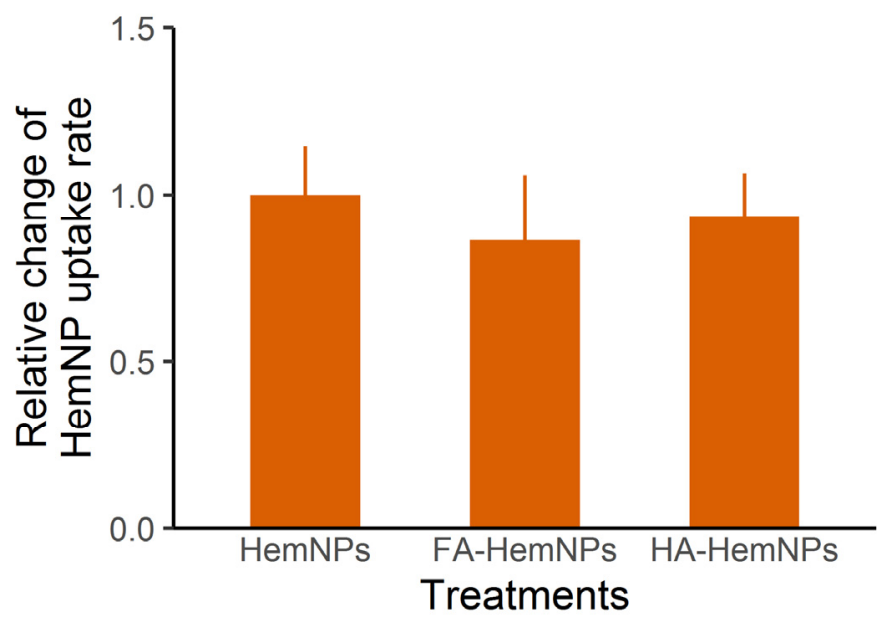

128 Figure S9. Effects of non-detectable adsorption of NOM on HemNP uptake. The uptake

129 rate of the HemNPs exposed to FA (FA-HemNPs) or HA (HA-HemNPs) before the uptake

130 experiment was comparable to that of raw HemNPs (HemNPs). No NOM was added to the

131 uptake medium. NOM-exposed HemNPs were separated from unbound NOM by asymmetric

132 flow field-flow fractionation. Data are presented as the mean \pm s.d. $(n=3)$.

133 


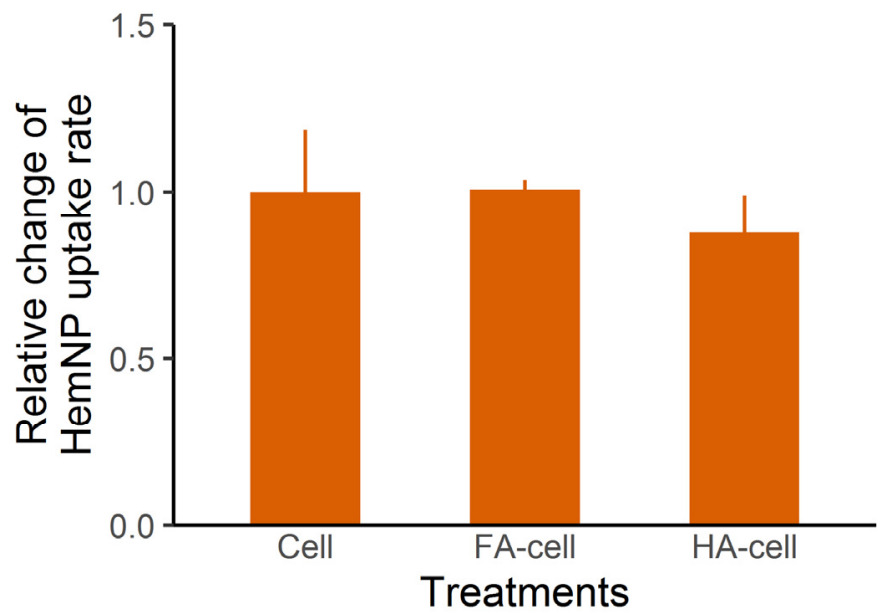

135 Figure S10. HemNP uptake by the cells pre-exposed to NOM. The rate of HemNP uptake

136 by T. thermophila was similar in cells pre-exposed (FA-cell or HA-cell) or not (Cell) to NOM.

137 Data are presented as the mean \pm s.d. $(n=3)$.

138 